phys. stat. sol. (b) 216, 775 (1999)

Subject classification: 68.55.Jk; 68.35.Gy; 78.30.Fs; S7.14

\title{
Strain Distribution in GaN Hexagons Measured by Raman Spectroscopy
}

\author{
R. Seitz ${ }^{1}$ ) (a), T. Monteiro (a), E. Pereira (a), and M. Di Forte-Poisson (b) \\ (a) University of Aveiro, Aveiro, Portugal \\ (b) Thomson-CSF, Orsay, France
}

(Received July 4, 1999)

Epitaxial growth of GaN layers normally starts in a three-dimensional growth mode at nucleation sites. Growth islands are formed which coalesce when a certain layer thickness is achieved. In our samples these islands show hexagonal structure and in some cases a flat surface plane perpendicular to the (0001) growth direction. We studied these hexagons by spatially resolved Raman spectroscopy. Raman spectroscopy is a powerful tool to determine strain in GaN layers, because the $\mathrm{E}_{2}$ Raman modes are very sensitive to the change of the elastic properties of the material. In all cases we found that the centre of the hexagons is nearly strain free whereas at the edges there is a tensile strain. This tensile strain, however, is not as high as in the surrounding film.

\section{Introduction}

Epitaxial growth of GaN layers normally does not start with a two-dimensional growth but rather in a three-dimensional growth mode at nucleation sites. Crystals are formed which coalesce when a certain layer thickness is achieved. Only then one can suppose a two-dimensional growth mode. In layers without buffer layer or with a thin GaN buffer layer, we can find growth islands which have not yet coalesced. These islands have in general hexagonal structure and sometimes a flat surface plane perpendicular to the (0001) growth direction. These hexagons vary in size (10 to $100 \mu \mathrm{m})$ but show always the same symmetry indicating that there is no misorientation in the growth process but rather an inhomogeneous growth rate along the wafer.

\section{Materials and Methods}

Our samples were grown with a GaN buffer layer on the c-plane (0001) of sapphire substrates. In the so-called two-step MOCVD growth process an intermediate buffer layer is grown at low temperature $\left(480{ }^{\circ} \mathrm{C}\right)$ on the substrate, followed by the growth of the high quality $\mathrm{GaN}$ film at higher temperature $\left(960^{\circ} \mathrm{C}\right)$. We studied the strain distribution in the hexagons by spatially resolved Raman spectroscopy. Micro-Raman measurements were carried out with the $514.5 \mathrm{~nm}$ line of an Ar ion laser coupled to a microscope. The Raman signals were detected by a liquid nitrogen cooled CCD. We used a $100 \times$ objective which allows a spatial resolution of approximately $1 \mu \mathrm{m}$. Raman spectroscopy is a powerful tool to determine strain in crystals, because some of the active Raman modes are very sensitive to the change of the elastic properties of the

\footnotetext{
1) Corresponding author; Tel.: ++351 34 370200; Fax: ++351 34 24965; e-mail: seitz@fis.ua.pt
} 


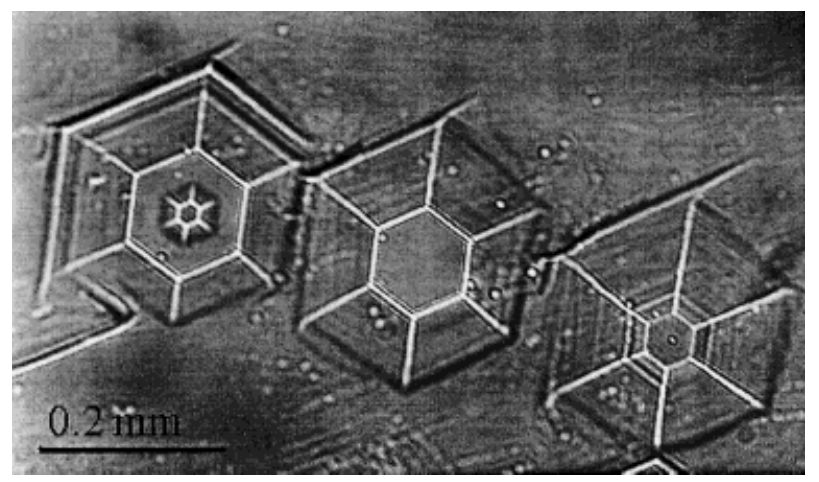

Fig. 1. Micrograph of GaN film showing typical hexagons

material. In $\mathrm{GaN}$ the $\mathrm{E}_{2}$ modes are very intense and sensitive to strain. Therefore we chose the $z(x, y) \underline{z}$ configuration where the $\mathrm{E}_{2}$ modes are allowed. The $z$-axis is along the (0001) growth direction. The peak position and FWHM of the Raman lines were determined by approximating the lineshapes with Lorentzian curves. The results were compared with values from a $7 \mu \mathrm{m}$ thick reference sample and from literature. Recent reports show that the $E_{2}$ phonon of a strain free sample has an energy of $568 \mathrm{~cm}^{-1}$ and that the shift of the $E_{2}$ phonon with biaxial strain is $4.1 \mathrm{~cm}^{-1} / \mathrm{GPa}$ [1]. Our reference sample shows an $E_{2}$ peak energy at $568.3 \mathrm{~cm}^{-1}$. The difference to the literature value is within the experimental error. Therefore we consider our reference sample as strain free.

\section{Results and Discussion}

The micrograph in Fig. 1 shows typical hexagons at different growth phases. Some of the hexagons show flat surface planes. We measured strain distribution in these planes.

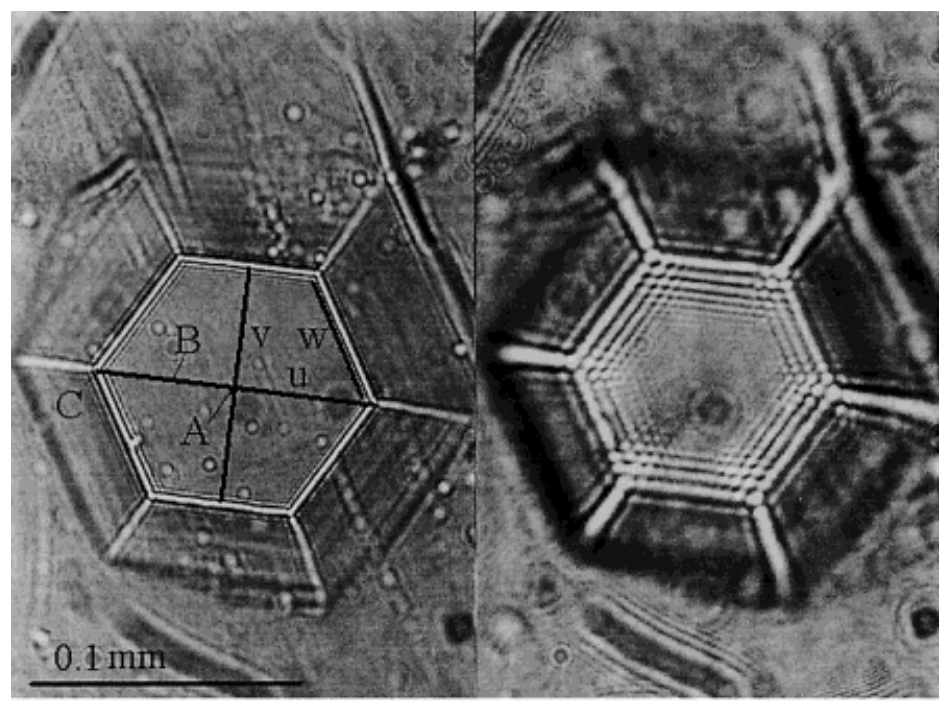

a)

b)

Fig. 2. Micrograph of hexagon a) without and b) with polarizer 


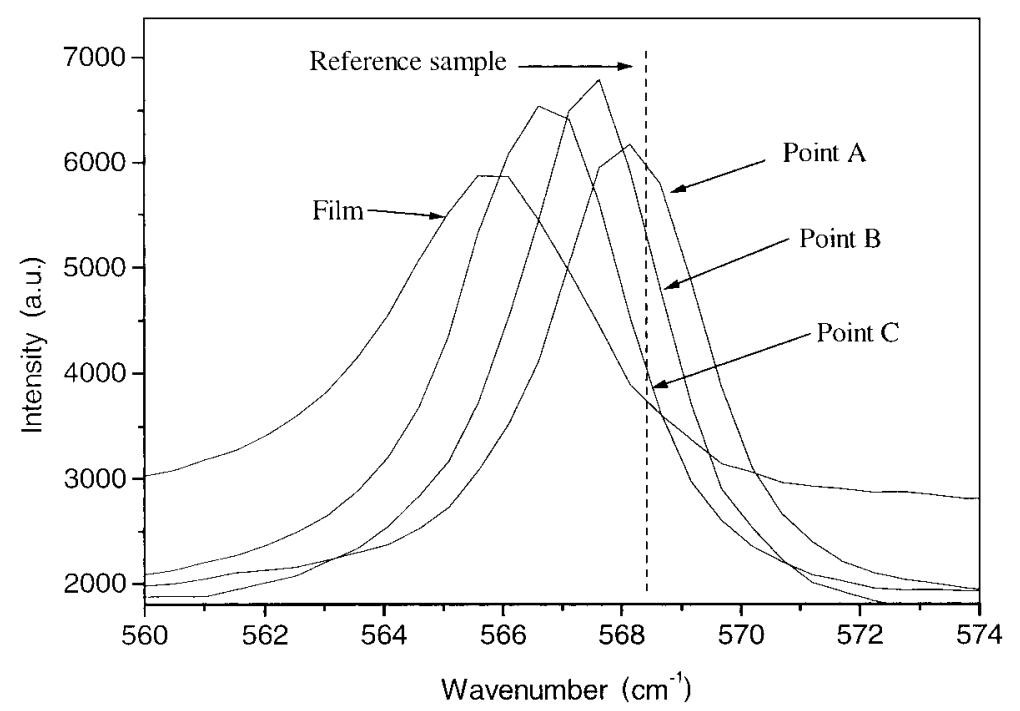

Fig. 3. Raman spectra along the $u$-axis of the hexagon of Fig. 2

Fig. 2 shows a microscope image of a hexagon taken with (part b) and without (part a) polarizers. The fringes seen in the micrograph taken with polarizers indicate strain in the surface plane of the hexagon. For a quantitive analysis of the strain, Raman spectra were taken along the indicated lines $(u, v, w)$. Fig. 3 shows the Raman spectra at three different points $(\mathrm{A}, \mathrm{B}, \mathrm{C})$ along the $u$-axis. The peak position of the $\mathrm{E}_{2}$ mode decreases monotonically when moving from the centre of the hexagon to the edge. Fig. 4 shows the $\mathrm{E}_{2}$ peak position profiles along the $u$ - and the $v$-axis. The peak position is constant when measured along the edge of the hexagon ( $w$-line).

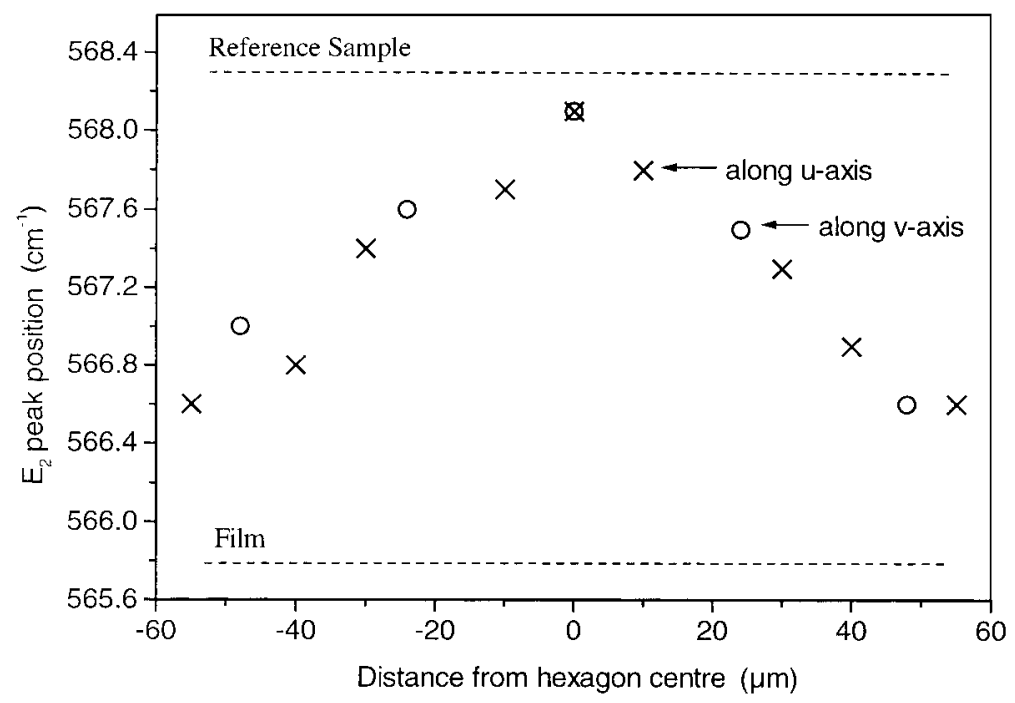

Fig. 4. $\mathrm{E}_{2}$ peak positions as a function of distance from the hexagon centre 
The film surrounding the hexagons shows significant tensile strain. This is very typical for our films [2]. However, it is in contrast to other reports [3] about GaN grown on sapphire. Since the peak position of the $E_{2}$ Raman line aproaches the strain free value of the reference sample, we can assume that the centre of the hexagons is strain free. Strain relaxation is usually due to the creation of dislocations. Several recent reports show that self-organized (overgrown) hexagonal pyramids have completely relaxed upper parts with centres that show a high density of impurities [4, 5]. However, their pyramids were selectively grown by using a $\mathrm{SiO}_{2}$ mask which could cause for example oxygen contamination, and the residual strain in their layers was compressive rather than tensile. Mao et al. found that GaN pyramids grown by selective lateral overgrowth show a very high density of structural defects [6]. Piqueras et al. studied "naturally" grown hexagonal-like topographic features of $\mathrm{GaN}$ : Si films [7]. They also found evidence for enhanced density of structural defects at the centres of the hillocks. Therefore we can assume that the strain relaxation in our samples is due to the formation of structural defects.

The tensile strain at the edges of the hexagons amounts to approximately $-0.41 \mathrm{GPa}$ according to Ager's relation between the shift of the $\mathrm{E}_{2}$ mode and residual strain. This value is still lower than the residual tensile strain in the surrounding film: $-0.61 \mathrm{GPa}$.

\section{Conclusion}

We found that hexagons which are a result of three-dimensional growth of GaN films on sapphire show a particular strain distribution. The centres of the hexagons are nearly strain free whereas at the edges tensile strain is found which is, however, not as high as in the surounding film. The complete strain relaxation in the centre of the hexagons could be explained by a higher density of dislocations. The understanding of the physical properties of structures like the hexagonal GaN pyramids is very important for possible future applications as shown in [8].

Acknowledgements One of the authors (R.S.) acknowledges financial support by FCT (Praxis XXI/BD/16284/98). The samples were provided in cooperation within the BriteEuram Project Rainbow (BRPR-CT96-0340).

\section{References}

[1] J. Ager, T. Suski, S. Ruvinov, J. Krueger, G. Conti, E. Weber, M. Bremser, R. Davis, and C. Kuo, Mater. Res. Soc. Symp. Proc. 449, 775 (1997).

[2] R. Seitz, T. Monteiro, E. Pereira, and M. di Forte-Poisson, phys. stat. sol. (a) 176, 661 (1999), this conference.

[3] W. Rieger, T. Metzger, H. Angerer, R. Dimitrov, O. Ambacher, and M. Stutzmann, Appl. Phys. Lett. 68, 970 (1996).

[4] A. Hoffmann, J. Christen, H. Siegle, F. Bertram, M. Schmidt, L. Eckey, A. Kaschner, C. Thomson, K. Hiramatsu, S. Kitamura, and N. Sawaki, J. Cryst. Growth 189/190, 630 (1998).

[5] K. Zeng, J. Lin, H. Jiang, and W. Yang, Appl. Phys. Lett. 74, 1227 (1999).

[6] Z. Mao, S. Mckernan, C. Carter, W. Yang, and S. McPherson, MRS Internet J. Nitride Semicond. Res. 4S1, G3.13 (1999).

[7] M. Zaldivar, P. FernándeZ, and J. Piqueras, J. Appl. Phys. 83, 1 (1998).

[8] S. Bidnyk, B. Little, Y. Cho, J. Krasinski, J. Song, W. Yang, and S. McPherson, MRS Internet J. Nitride Semicond. Res. 4S1, G6.48 (1999). 